\title{
Perceiving Spirals and Inside/Outside Relations by A Neural Oscillator Network
}

\author{
Ke Chen and DeLiang L. Wang \\ Department of Computer and Information Science and Center for Cognitive Science \\ The Ohio State University, Columbus, OH 43210-1277, USA \\ E-mail: $\{$ kchen,dwang $\} @$ cis.ohio-state.edu
}

\begin{abstract}
Since first proposed by Minsky and Papert (1969), the spiral problem is well-known in neural networks. It receives much attention as a benchmark for various learning algorithms. Unlike previous work that emphasizes learning, we approach the problem from a generic perspective that does not involve learning. We point out that the spiral problem is intrinsically connected to the inside/outside problem. We propose a solution to both problems based on oscillatory correlation using a time delay network. Our simulation results match human performance, and we interpret human limitations in terms of synchrony and time delays, both biologically plausible. As a special case, our network without time delays can always distinguish these figures regardless of shape, position, size, and orientation. We conjecture that visual perception will be effortful if local activation cannot be rapidly propagated, as synchrony would not be established in the presence of time delays.
\end{abstract}

\section{INTRODUCTION}

The spiral problem refers to distinguishing between a connected single spiral and disconnected double spirals on a 2-D plane, as illustrated in Fig. 1. Since Minsky and Papert (1969) first introduced the problem in their influential book on perceptrons [1], it has received much attention from several different fields. In the neural network community, a variant of the problem has become a benchmark. Many papers have dealt with the problem and many solutions have been attempted using different learning models [3][4]. However, resulting learning systems are only able to produce decision regions highly constrained by the spirals defined in a training set, thus specific in shape, position, size, orientation etc. Furthermore, no cxplanation is provided as to why the problem is difficult for human subjects to solve. On the other hand, the problem is considered as an exceptional instance of figure-ground separation or anomalous perceptual stimuli in perceptual psychology since unlike most of visual stimuli the immediate perception cannot be achieved by humans [5]. Grossberg and Wyse proposed a neural network architecture for figure-ground separation of connected scenic figures and reported their architecture can distinguish between connected and disconnected spirals [6]. In their paper, however, no demonstration was given to the spiral problem, and their model does not exhibit the limitations that humans do.

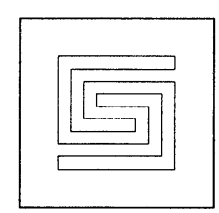

(a)

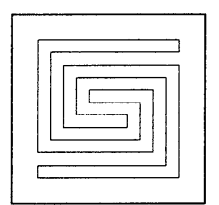

(b)
Fig. 1. The spiral problem. (a) a connected single spiral. (b) disconnected double spirals (adapted from [1][2]).

Ke Chen is on leave from National Laboratory of Machine Perception, Peking University, Beijing 100871, China
There is a related problem in the study of visual perception, i.e., the perception of inside/outside relations. Considering the visual input of a single closed curve, the task of perceiving the inside/outside relation is to determine whether a specific pixel lies inside or outside the closed curve. For the human visual system, the perception of inside/outside relations often appears to be immediate and effortless. However, the immediate perception is not available for humans when the bounding contour becomes highly convoluted [7][8]. Ullman (1984) suggested the computation of spatial relation through the use of visual routines. Visual routines result in the conjecture that the inside/outside is inherently sequential. As pointed out recently by Ullman, the processes underlying the perception of inside/outside relations are as yet unknown and applying visual routines is simply one alternative [8].

There are many cortex areas in the mammalian visual system and an everyday object will excite cells in most of these area. In vision, therefore, binding is viewed as a way of integrating fragmentary neural events at multiple locations in the brain to produce unified perceptual experience and behavior. Theoretical investigations of brain functions indicate that timing of ncuronal activity is a key to the construction of neuronal assemblies [9][10]. In particular, a binding mechanism that uses neural oscillations to encode temporal correlation has received considerable support biologically [11]. The discovery of synchronous oscillations in the visual cortex has triggered much interest to develop computational models for oscillatory correlation. Recently, Terman and Wang proposed locally excitatory globally inhibitory oscillator networks (LEGION) [12][13]. They theoretically showed that LEGION can rapidly achieve both synchronization in a locally coupled oscillator group representing each object and desynchronization among a number of oscillator groups representing multiple simultaneously presented objects. In particular, synchrony in LEGION is achieved at an exponential rate. More recently, Campbell and Wang have studied time delays in networks of relaxation oscillators and analyzed the behavior of $\mathrm{LE}$ GION with time delays [14]. Their studies show that loosely synchronous solutions can be achieved under a broad range of initial conditions and time delays. Therefore, LEGION provides a computational framework to study the process of visual perception from a standpoint of oscillatory correlation.

We explore both the spiral problem and the inside/outside problem by oscillatory correlation in this paper. We attempt to address the following problems: Why humans cannot immediately distinguish between connected and disconnected 
spirals? What is a connection between the spiral problem and the inside/outside relation? How to perceive the inside/outside relation in neural networks? We show that computation through LEGION with time delays yields a genetic solution to these problems. This investigation leads to the interpretation that perceptual performance would be limited if local activation cannot be rapidly propagated due to time delays. As a special case, we demonstrate that LEGION without time delays reliably distinguishes between connected and disconnected spirals and discriminates the inside and the outside regardless of shape, position, size, and orientation. Here, we emphasize that there is no learning process in LEGION and suggest that this kind of problems may be better solved by a properly connected architecture with oscillatory dynamics rather than by sophisticated learning.

\section{Methodology}

The architecture of LEGION used in this paper is a 2D network. Each oscillator is connected to its four nearest neighbors, and the global inhibitor receives excitation from each oscillator on the network and in turn inhibits each oscillator [12][13]. In LEGION, a single oscillator $i$, is defined as a feedback loop between an excitatory unit $x_{i}$ and an inhibitory unit $y_{i}$ :

$$
\begin{gathered}
\frac{d x_{i}}{d t}=f\left(x_{i}, y_{i}\right)+I_{i}+S_{i}+\rho \\
\frac{d y_{i}}{d t}=c g\left(x_{i}, y_{i}\right)
\end{gathered}
$$

where $f\left(x_{i}, y_{i}\right)=3 x_{i}-x_{i}^{3}-y_{i}$, and $g\left(x_{i}, y_{i}\right)=\lambda+$ $\gamma \tanh \left(\beta x_{i}\right)-y_{i}$ are used in this paper. $I_{i}$ represents external stimulation to the oscillator, and $S_{i}$ represents overall coupling from other oscillators in the network. The symbol $\rho$ denotes the amplitude of a Gaussian noise. Other parameters $\epsilon, \beta, \lambda$ and $\gamma$ are chosen to control a periodic solution of the dynamic system. The periodic solution alternates between silent and active phases of near steady-state behavior [12][13]. The coupling term $S_{i}$ at time $t$ is

$$
S_{i}=\sum_{k \in N(i)} W_{i k} S_{\infty}\left(x_{k}(t-\tau), \theta_{x}\right)-W_{z} S_{\infty}\left(z, \theta_{z}\right)
$$

where $S_{\infty}(x, \theta)=1 /(1+\exp [-\kappa(x-\theta)])$ and the parameter $\kappa$ controls the steepness of the sigmoid function. $W_{i k}$ is a synaptic weight from oscillator $k$ to oscillator $i$, and $N(i)$ is the set of its immediate neighbors. $\tau(\tau \geq 0)$ is a time delay in interactions [14], and, in particular, there is no time delay when $\tau=0 . \theta_{x}$ is a threshold over which an oscillator can affect its neighbors. $W_{z}$ is the positive weight used for inhibition from the global inhibitor $z$, whose activity is defined as

$$
\frac{d z}{d t}=\phi\left(\sigma_{\infty}-z\right)
$$

where $\sigma_{\infty} \equiv 0$ if $x_{i}<\theta_{z}$ for every oscillator $i$, and $\sigma_{\infty} \equiv 1$ if $x_{i}(l) \geq \theta_{z}$ for at least one oscillator $i$. Here $\theta_{z}$ represents a threshold to determine whether the global inhibitor $z$ sends inhibition to oscillators, and the parameter $\phi$ determines the rate at which the inhibitor reacts to stimulation from oscillators. The computation of LEGION can be briefly summarized as follows. Once an oscillator is in the active phase, it triggers the global inhibitor. This then inhibits the entire network. On the other hand, an active oscillator propagates its activation to its nearest neighbors, again through Equation 2, and from them to its further neighbors. Thus, the entire dynamics of LEGION is a combination of local cooperation through excitatory coupling among neighboring oscillators and global competition via the global inhibitor.

For a given image consisting of $N \times N$ pixels, a 2-D LEGION network with $N \times N$ oscillators is employed so that each oscillator in the network corresponds to a pixel in the image. In this paper, all images are binary to be consistent with typical stimuli used in the original problems. As a convention, black pixels correspond to either figure or background and white pixels correspond to boundaries separating figures and the background in general. When figures are colored into black ones, however, black pixels will correspond to figures and white pixels will correspond to backgrounds. In simulations, we let the external input of an oscillator representing any pixel in figures or background be positive, and the external input of oscillators representing boundaries be negative accordingly. An effective connection is built between two oscillators if and only if they are neighbors and both of them are activated by external stimulation. Note that boundary conditions may cause some distortion to the stability of synchronous oscillations. Therefore, we also adopt the dynamic normalization technique [12][13] so that the weights of all effective connections to one stimulated oscillator are normalized to a constant.

We use pattern formation in oscillator networks to refer to the behavior that all the oscillators representing the same object are synchronous, while the oscillators representing different objects are desynchronous. Terman and Wang have analytically shown that such a solution can be achieved in LEGION without time delays [13]. However, a solution may not be achieved when time delays are introduced. Although the loose synchrony concept has been introduced to describe time delay behavior [14], it does not indicate pattern formation in an entire network even when loose synchrony is achieved because loose synchrony is a local concept defined in terms of pairs of neighboring oscillators. Here we introduce a measure called min-max difference in order to examine whether pattern formation is achieved. Suppose that oscillators $O_{i}$ and $O_{j}$ represent two pixels in the same object, and the oscillator $O_{k}$ represents a pixel in a different object. Moreover, let $t^{s}$ denote the time at which oscillator $O_{s}$ enters the active phase. The min-max difference measure is defined as

$$
\left|t^{i}-t^{j}\right|<\tau_{R B} \text { and }\left|t^{i}-t^{k}\right| \geq \tau_{R B}
$$

where $\tau_{R B}$ is the time period of an active phase. Intuitively, this measure suggests that pattern formation is achieved if any two oscillators representing two pixels in the same object have some overlap in the active phase, while any two oscillators representing two pixels belonging to different objects never stay in the active phase simultaneously. This definition of pattern formation applies to both exact synchrony in LEGION without time delays and loose synchrony with time delays. As a result, pattern formation is achieved by a LEGION network within $P \times T$ where $T$ is the period 
of oscillation which can be calculated explicitly in terms of system parameters and $P$ is the number of objects in the presented image. This statement is based on the theoretical result [13].

We adopt two methods to display our simulation results. One is the combined $x$ activities of the oscillators representing the same object as well as the global inhibitor over time, and the other is snapshots to show instantaneous activity of the entire network at various stages of dynamic evolution. When pattern formation occurs, there will always be an overlapping time when all the oscillators representing the same object are in the active phase, while there will be no time when two oscillators representing two pixels belonging to different objects are in the active phase simultaneously. For snapshots, we use black circles to show $x$ activities of oscillators and stipulate that the diameter of a black circle is proportional to $\left(x-x_{\min }\right) /\left(x_{\max }-x_{\min }\right)$. Here we assume that $x$ values of all oscillators lie in the interval from $x_{\text {min }}$ to $x_{\text {max }}$.

\section{Simulations}

In the following simulations, the differential equations in Equations 1-3 were numerically solved using the fourthorder Runge-Kutta method. We illustrate stimulated oscillators with black squares. All oscillators were initialized randomly. To explore genetic solutions from a biologically plausible standpoint, it is desirable to use LEGION with time delays because time delays inevitably occur in information transmission of a biological system. The performance of LEGION without time delays is shown as a special case to illustrate its capabilities of solving problems. A large number of simulations have been conducted [15], and similar results as reported here are produced with a broad range of parameter values and network sizes. Here we report typical results using a specific set of parameter values.

\section{A. The Spiral Problem}

As illustrated in Fig. 1, the spiral problem consists of two images proposed by Minsky and Papert [1][2]. In Fig. 1(a), it is a connected single spiral, while Fig. 1(b) contains disconnected double spirals. For simulations, they were sampled as two binary images with $29 \times 29$ pixels. For these images, two problems can be asked as follows: (1) When an image is presented, can one determine whether it contains a single spiral or double spirals? (2) Given a point on a 2-D plane, can one determine whether it is inside or outside a specific spiral?

We first applied LEGION with time delays to the single spiral image, and Fig. 2 shows the results. Fig. 2(a) illustrates the visual stimulus, where black pixels represent either the spiral or the background and white pixels represent the boundary between the two. In other words, black pixels correspond to stimulated oscillators and white ones unstimulated oscillators. Fig. 2(b) shows a sequence of snapshots after the network was stabilized except for the first snapshot which shows the random initial state of the network. A snapshot represents network activity at a particular time instance. These snapshots are arranged in temporal order first from left to right and then from top to bottom. Fig. 2(c) shows the temporal trajectories of the combined $x$ activities of the oscillators representing the spiral and the background as well as the temporal activity of the global inhibitor. We observe from these snapshots that an activated oscillator in the spiral propagates its activation to its two immediate neighbors with some time delay, and the process of propagation forms a traveling wave along the spiral. We emphasize that, at any time, only the oscillators corresponding to a portion of the spiral stay in the active phase together, and the entire spiral can never be in the active phase simultaneously. Thus, based on the oscillatory correlation theory, our system cannot group the whole spiral together, and it fails to realize that the pixels in the spiral belong to the same pattern. The system's limitation matches that of human performance in this situation. Also, the process that our model traces the spiral appears similar to the way humans sequentially trace the spiral for identification. Note that the convoluted part of the background behaves similarly. According to the min-max difference measure, Fig. 2(c) shows that pattern formation cannot be achieved.

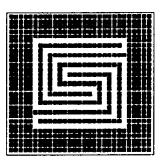

(a)
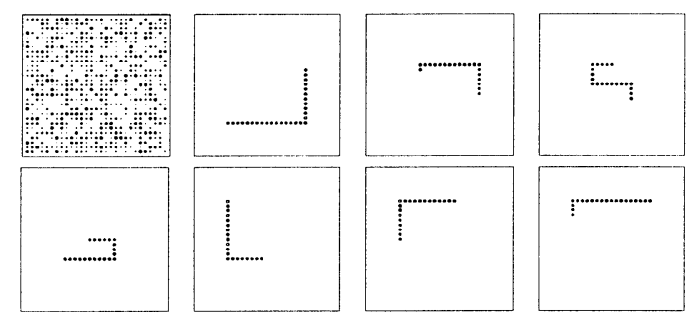

(b)

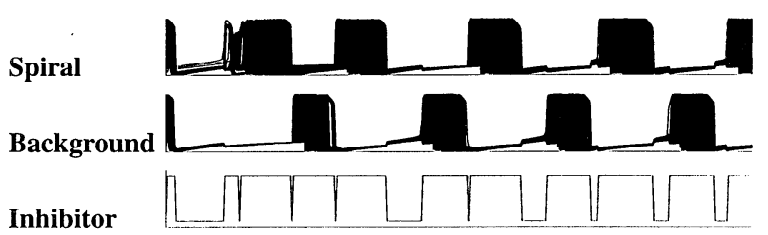

(c)

Fig. 2. Results of LEGION with a time delay $\tau=0.002 T$ ( $T$ is the period of oscillation) to the spiral problem. The parameter values used in this simulation are $\epsilon=0.003, \beta=500, \gamma=21.0, \lambda=21.5, \alpha_{T}=6.0$, $\rho=0.03, \kappa=500, \theta_{x}=-0.5, \theta_{z}=0.1, \phi=3.0, W_{z}=1.5$, $I_{s}=1.0$, and $I_{u}=-1.0$ where $I_{s}$ and $I_{u}$ are external input to stimulated and unstimulated oscillators, respectively.

In order to illustrate the effects of time delays, we applied LEGION without time delays to the same image, and Fig. 3 shows the results. Fig. 3(a) illustrates the same visual stimulus. Fig. 3(b) shows three snapshots corresponding to the random initial state of the network, the separated spiral and the background, respectively. Fig. 3(c) illustrates the temporal trajectories of the stimulated oscillators and the global inhibitor. It is evident from Fig. 3 that pattern formation is achieved, and the single spiral can be segregated from the background by the second period. Thus, LEGION without time delays can readily solve the spiral problem in this case. The failure to group the spiral in Fig. 3 is caused by time delays in the coupling of neighboring oscillators. 


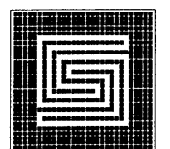

(a)
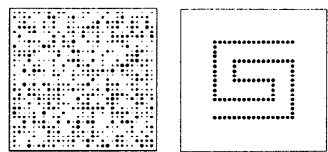

(b)

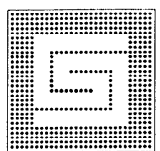

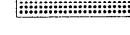

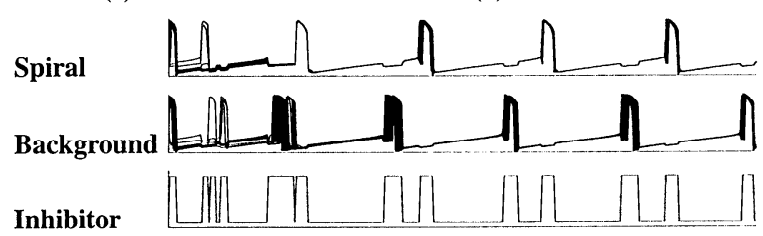

(c)

Fig. 3. Results of LEGION without time delays to the spiral problem. The parameter values used are the same as listed in the caption of Fig. 2.

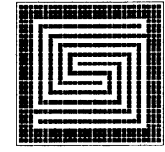

(a)
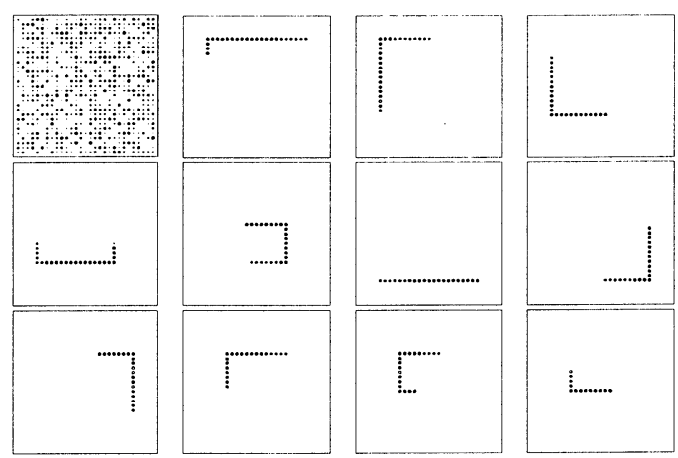

(b)

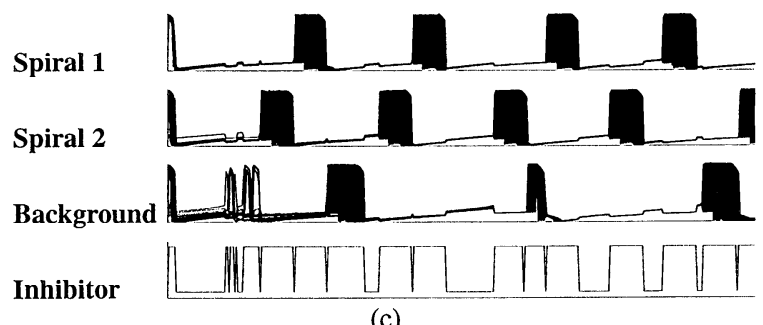

(c)

Fig. 4. Results of LEGION without time delays to the spiral problem. The parameter values used are the same as listed in the caption of Fig. 2.

We also applied LEGION with time delays to the double spirals image. Fig. 4 shows the results. Fig. 4(a) shows the visual stimulus. Fig. 4(b) shows a sequence of snapshots arranged in the same order as in Fig. 2(b). We observe from these snapshots that starting from an end of one spiral a traveling wave is formed along the spiral and the activated oscillators representing the spiral propagate their activation. Due to time delays, however, only the oscillators corresponding to a portion of the spiral stay in the active phase together, and the entire spiral is never in the active phase simultaneously. The oscillators representing the other spiral have the same behavior. The results show that the pixels in any one of double spirals cannot be grouped as the same pattern. Again, the system's limitation is consistent with that of human performance in this situation. Also, the process that our model traces a spiral appears to resemble the way human spiral tracking for identification. We mention that the behavior of our system for the convoluted part of the background is similar to that for the double spirals. Fig. 4(c) illustrated the temporal trajectories of activities of all the stimulated oscillators and the global inhibitor. Note that our further observation beyond the duration of Fig. 4(c) shows that the system is stabilized by the fourth period of oscillations. The temporal trajectories of the spirals and the background indicates that pattern formation is not achieved after the network was stabilized. We also applied LEGION without time delays to the double spirals image for the same purpose as described before. Simulation results also show that one spiral can be segregated from both the other spiral and the background by the second period [15]. Thus, LEGION without time delays readily solves the spiral problem in this case, and it indicates that the failure to group the double spirals in Fig. 4 results from time delays.

For the spiral problem, pattern formation means that solutions in the aforementioned two problems can be provided to the questions of counting the number of objects or identifying whether two pixels belong to the same spiral or not. No such solutions are available when pattern formation is not achieved. Hence, our system cannot solve the spiral problem in general. Only under the special condition of no time delay can our system solve the problem.

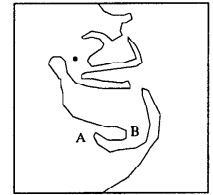

(a)

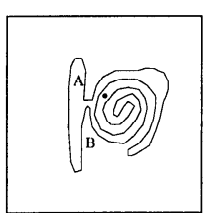

(b)
Fig. 5. Inside/outside relations. (a) an example where immediate perception is available (adapted from [16]). (b) an example where immediate perception is not available (adapted from [7]).

\section{B. Inside/Outside Relations}

The inside/outside problem refers to whether a dot belongs to area A or to area B, as illustrated in Fig. 5. The perception of inside/outside relations is usually performed by humans with intriguing efficiency. Fig. 5(a) is such an example, where humans can effortlessly recognize the dot is inside area A rather than area B though the two areas are separated by a convoluted boundary. However, immediate perception is subject to limitations. For instance, humans cannot immediately identify whether the dot is inside area A or area B for the visual stimulus in Fig. 5(b). Here, we report our typical results produced by LEGION. For simulations, the two pictures were sampled as binary images with $43 \times 43$ pixels.

We first applied LEGION with time delays to the two images in Fig. 5, and Figures 6 and 7 show the results. Figures 6(a) and 7(a) show the visual stimuli, where black pixels represent areas $\mathrm{A}$ and $\mathrm{B}$ that correspond to stimulated oscillators and white pixels represent the boundary that corresponds to unstimulated oscillators. Figures 6(b) and 7(b) illustrate a sequence of snapshots after networks were stabilized except for the first snapshot which shows the random initial states of networks. Figures 6(c) and 7(c) show temporal trajectories of the combined $x$ activities of the oscillators representing areas $\mathrm{A}$ and $\mathrm{B}$ as well as the global inhibitor, 
respectively.

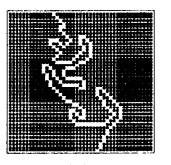

(a)

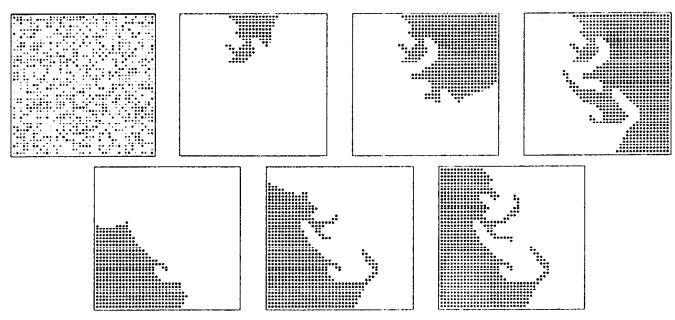

(b)

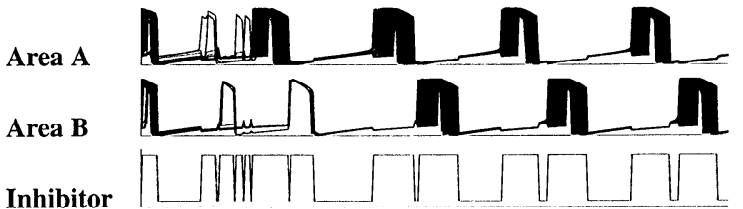

(c)

Fig. 6. Results of LEGION with a time delay $\tau=0.002 T$ to Fig. 5(a). The parameter values used in this simulation are $\epsilon=0.004, \gamma=14.0$, $\lambda=11.5$ and the other parameter values are the same as listed in the caption of Fig. 2.

We observe from Fig. 6(b) that the activation of an oscillator can rapidly propagate through its neighbors to other oscillators representing the same area, and eventually all the oscillators representing the same area (A or B) stay together in the active phase simultaneously, though they generally enter the active phase at different times due to time delays. Thus, on the basis of oscillatory correlation, our system can group an entire area (A or B) together and recognize all the pixels in area $\mathrm{A}$ or $\mathrm{B}$ as elements of the same area. According to the min-max difference measure, moreover, Fig. 6(c) shows that pattern formation is achieved by the second period. In contrast, we observe from Fig. 7(b) that although an activated oscillator rapidly propagates its activation in open regions as shown in the last three snapshots, propagation is limited once the traveling wave propagates in spiral-like regions as shown in earlier snapshots. As a result, at any time, only the oscillators corresponding to a portion of either area stay in the active phase together, and the oscillators representing the whole area are never in the active phase simultaneously. On the basis of oscillatory correlation, our system cannot group the whole area, and fails to identify the pixels of one area as belonging to the same pattern. Again, this limitation of our system matches that of human performance in this situation. Furthermore, according to the min-max difference measure, Fig. 7(c) shows that pattern formation is not achieved after the network was stabilized.

In order to illustrate the effects of time delays and show how to use an oscillator network to perceive inside/outside relations, we applied LEGION without time delays to the two images in Fig. 5. Our simulations show that LEGION without time delays readily segregates two areas in both cases by the second period [15]. Thus, the failure to group each area in Fig. 7 is attributed to time delays in the coupling of neighboring oscillators.

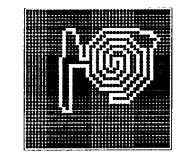

(a)
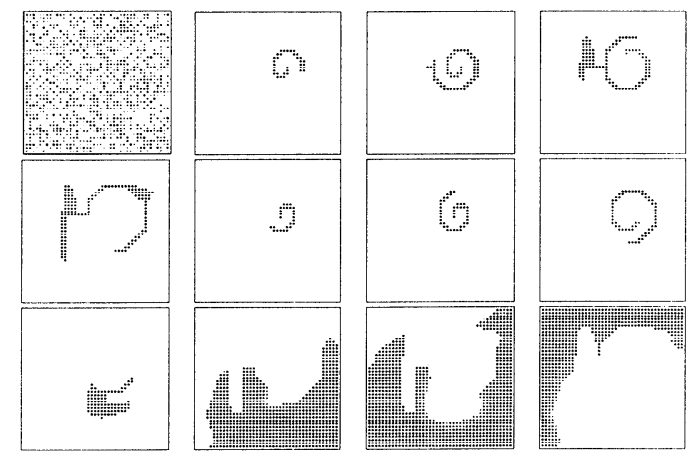

(b)

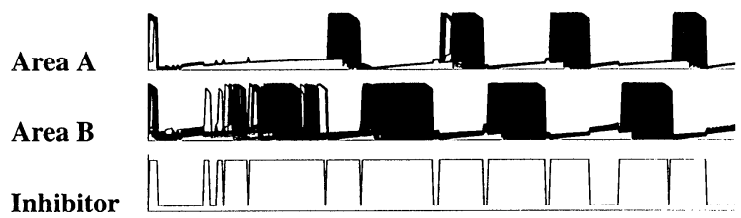

(c)

Fig. 7. Results of LEGION with a time delay $\tau=0.002 T$ to Fig. 5(b). The parameter values used are the same as listed in the caption of Fig. 6.

In general, the above simulations suggest that oscillatory correlation provides a way to address inside/outside relations by a neural network; when pattern formation is achieved, a single area segregates from other areas that appear in the same image. For a specific dot on the 2-D plane, the inside/outside relations can be identified by examining whether the oscillator representing the dot synchronizes with the oscillators representing a specific area or not.

\section{DISCUSSION AND CONCLUSION}

The relaxation oscillator, a component of LEGION, is dynamically very similar to numerous other oscillators used in modeling neuronal behavior, while oscillatory correlation is consistent with grouping evidence that supports the existence of neural oscillation in the visual cortex and other brain regions. Furthermore, the local excitatory connections adopted in the network are motivated by various lateral connections in the brain, in particular, the horizontal connections in the visual cortex [17]. The global inhibitor on the other hand, might be viewed as a neuronal group in the thalamus, and its activity may be interpreted as the collective behavior of the neuronal group. On the other hand, recent theoretical studies on perceptual organization show that the formation of uniform and connected regions is probably the first step in perceptual organization [18]. This suggestion agrees with the emphasis on emergent synchrony based on local connectivity, which reflects both connectedness and uniformity. The binding mechanism in LEGION provides a neurocomputational foundation to generate uniform and 
connected regions. Physiological studies also show that there are waves of neural activity traveling with velocities of $10-90 \mathrm{~cm} / \mathrm{sec}$ in the hippocampus and other cortical areas. On the other hand, the speed of nerve conduction is less than $1 \mathrm{~m} / \mathrm{sec}$ in local cortical circuits [19] such that connected neurons with $1 \mathrm{~mm}$ apart have a time delay of more than $4 \%$ of the period of oscillation assuming $40 \mathrm{~Hz}$ oscillations. Therefore, the use of LEGION with time delays represents a general case, while the use of LEGION without time delays should be regarded as a special case. The biological plausibility of our system allows us to interpret its computation as a visual perceptual process.

As a benchmark task, a variant of the spiral problem is well known in the neural network community. Although it has been reported that many neural network models can solve the problem through learning, their solutions are subject to limitations because generalization abilities of resulting learning systems highly depend on the training set. When slightly different spirals (e.g., shape, position, size, and orientation) are used as input to learned systems, the correct solution cannot be generated. As pointed out by Minsky and Papert [1][2], solving the spiral problem is equivalent to detecting connectedness. From the viewpoint of computational geometry, Minsky and Papert showed that connectedness cannot be computed by any diameter-limited or order-limited perceptrons [1]. This limitation holds for multi-layered perceptrons regardless of learning scheme ([2], p.252). Unfortunately, few people have discussed generality of their solutions. In contrast, LEGION provides a genetic solution to the spiral problem. Our simulations have shown that LEGION without time delays can always distinguish these figures regardless of shape, position, size and orientation. In particular, we emphasize that no learning is involved in LEGION. Therefore, we suggest that the spiral problem may be better solved by a network of oscillators without learning.

The perception of inside/outside relation involves a complicated procedure of visual information processing beyond early representations [7][8]. Ullman suggested that the related computation consists of two main stages. The first stage is the bottom-up creation of certain representations of the visual environment. The second stage involves serial applications of processes called visual routines on the representations constructed in the first stage. These visual routines can establish properties and relations which cannot be represented explicitly in the initial representation. For perceiving inside/outside relations, a so-called coloring method was proposed [7] and can be summarized as follows. Starting from a given point, its surrounding area in the internal representation is activated. The activation stretches out until a boundary is reached. Depending on the starting point, either the inside or the outside of the boundary will be activated, but simultaneous activation of both is not allowed. The coloring scheme continues to operate until the activation cannot spread. This provides a basis of separating the inside from the outside. Our simulation results show that activity propagation in LEGION with time delays forms a traveling wave within a specific object. When an object is a spiral-like structure as in Fig. 5(b), activation spreads slowly, while activation spreads rapidly when the object is a wide open structure. This behavior of LEGION is similar to the process of the coloring scheme. However, there are a number of important differences. First, the coloring method is described as a serial algorithm, while our system is an inherently parallel and distributed process though its emergent behavior reflects a degree of serial nature of the problems. Second, the coloring method does not make a qualitative distinction between rapid effortless perception that corresponds to simple boundaries and slow effortful perception that corresponds to convoluted boundaries - the time the coloring routine takes varies continuously. In contrast, our system makes such a distinction: effortless perception with simple boundaries corresponds to when pattern formation is achieved, and effortful perception with convoluted boundaries corresponds to when pattern formation is not achieved. Third, perhaps more importantly conceptually, our systern does not invoke high-level serial process to solve such problems like inside/outside relations; its solution involves the same mechanism as it does for parallel image segmentation (see [20]).

\section{ACKNOWLEDGMENTS}

Authors arc grateful to S. Campbell for many discussions. This work was supported in part by an NSF grant (IRI-9423312), an ONR grant (N00014-93-10335) and an ONR Young Investigator Award (N00014-961-0676) to DLW.

\section{REFERENCES}

[1] M. Minsky and R. Papert, Perceptrons, MIT Press, 1969.

[2] M. Minsky and R. Papert, Perceptrons (extended version), MIT Press, 1988

[3] K. Lang and M. Witbrock, "Learning to tell two spirals apart," Proc. 1988 Connectionist Models Summer School, Morgan Kaufmann, 1988, 52-59.

[4] S. Fahlman and C. Lebiere, "The cascade-correlation learning architecture," NIPS-2, Morgan Kaufmann, 1990, 524-532.

[5] I. Howard, "Proposals for the study of anomalous perceptual schemata," Perception 3, 1974, 497-513.

[6] S. Grossberg and L. Wyse, "A neural network architecture for figure-ground separation of connected scenic figures," Neural Net. 4, 1991, 723-742.

[7] S. Ullman, "Visual routines," Cognition 18, 1984, 97-159.

[8] S. Ullman, High-level vision, MIT Press, 1996.

[9] P. Milner, "A model for visual shape recognition," Psychol. Rev. 81 $1974,512-535$.

[10] von der Malsburg, "The correlation theory of brain function," Internal Report 81-2, Max-Planck-Institute for Biophysical Chemistry, 1981.

[11] W. Singer and C. M. Gray, "Visual feature integration and the temporal correlation hypothesis," Ann. Rev. Neurosci. 18, 1995, 555586.

$[12\rceil$ D. L. Wang and D. Terman, "Locally excitatory globally inhibitory oscillator networks," IEEE Trans. Neural Net. 6, 1995, 283-286.

[13] D. Terman and D. L. Wang, "Global competition and local cooperation in a network of neural oscillators," Physica D 81, 1995, 148 176.

[14] S. Campbell and D. L. Wang, "Relaxation oscillators with time delay coupling," Physica $D, 1997$. (in press)

[15] K. Chen and D. L. Wang, "Perceiving without learning: from spirals to inside/outside relations," Technical Report OSU-CISRC-8/97TR38, The Ohio State University, 1997.

[16] B. Julesz, Dialogues on perception, MIT Press, 1995.

[17] C. D. Gilbert, "Horizontal integration and cortical dynamics," Neuron 9, 1992, 1-13.

[18] S. Palmer and I. Rock, "Rethinking perceptual organization: the role of uniform connectedness," Psychol. Bull. Rev. 1, 1994, 29-55.

[19] T. Murakoshi et al, "Electrophysiological identification of horizontal synaptic connections in rat visual cortex in vitro," Neurosci. Lett. 163, 1993, $211-214$.

[20] D. L. Wang and D. Terman, "Image segmentation based on oscillatory correlation," Neural Comp. 9, 1997, 805-836. 